\title{
A new treatment of mixed virtual and real IR-singularities
}

\section{Janusz Gluza}

Department of Field Theory and Particle Physics, Institute of Physics, University of Silesia, Uniwersytecka 4, PL-40-007 Katowice, Poland

E-mail: gluza@us.edu.pl

\section{Tord Riemann*}

Deutsches Elektronen-Synchrotron, DESY, Platanenallee 6, 15738 Zeuthen, Germany

E-mail: Tord.Riemannedesy.de

We discuss the determination of the infrared singularities of massive one-loop 5-point functions with Mellin-Barnes (MB) representations. Massless internal lines may lead to poles in the $\varepsilon$ expansion of the Feynman diagram, while unresolved massless final state particles give endpoint singularities of the phase space integrals. MB integrals are an elegant tool for their common treatment. An evaluation by taking residues leads to inverse binomial sums.

8th International Symposium on Radiative Corrections (RADCOR)

October 1-5 2007

Florence, Italy

${ }^{*}$ Speaker. 


\section{Introduction}

We study the infrared (IR) singularities of some massive one-loop $n$-point Feynman integrals,

$$
I=\frac{e^{\varepsilon \gamma_{E}}}{i \pi^{d / 2}} \int \frac{d^{d} k T(k)}{\left(q_{1}^{2}-m_{1}^{2}\right)^{v_{1}} \ldots\left(q_{i}^{2}-m_{i}^{2}\right)^{v_{j}} \ldots\left(q_{n}^{2}-m_{n}^{2}\right)^{v_{n}}},
$$

by representing them with standard Feynman parameter integrals with characteristic $F$ and $U$ forms:

$$
I=\frac{e^{\varepsilon \gamma_{E}}(-1)^{N_{v}} \Gamma\left(N_{v}-\frac{d}{2}\right)}{\prod_{i=1}^{n} \Gamma\left(v_{i}\right)} \int_{0}^{1} \prod_{j=1}^{n} d x_{j} x_{j}^{v_{j}-1} \delta\left(1-\sum_{i=1}^{n} x_{i}\right) \frac{U(x)^{N_{v}-d}}{F(x)^{N_{v}-d / 2}} P(T),
$$

with $N_{v}=\sum_{i=1}^{n} v_{i}$. For one loop integrals, the $U=\sum x_{i}$ may be set to one. The $F$-form is bilinear in the $x_{i}$ and may be represented in turn by a multiple Mellin-Barnes (MB) integral, using the representation:

$$
\frac{1}{(A(x)+B(x))^{v}}=\frac{1}{2 \pi i} \int_{-i \infty+R}^{i \infty+R} d z A(x)^{z} B(x)^{-v-z} \frac{\Gamma(-z) \Gamma(v+z)}{\Gamma(v)},
$$

where the integration contour separates the poles of the $\Gamma$-functions.

Afterwards, the Feynman parameters may be integrated out and one has to solve the resulting MB integral. This is in general quite non-trivial. However, there is an interesting kind of problems where a systematic approach might be developed, namely the evaluation of the IR divergent parts of the Feynman integrals. They are at the begin of the $\varepsilon$-expansion $(\varepsilon=(4-d) / 2)$ of the Feynman integral and so of smaller dimensionality in the variables $z$. In fact, usually one subtracts them from the rest of the integral and treats them separately.

The MB representation allows to do this in a special way which might be of some practical usefulness. We will discuss here only scalar one-loop functions, $T(k)=1$, but tensors don't show additional problems. For basic definitions and formulae we refer to [1]-3] and references cited therein. We use here and in the following the Mathematica packages AMBRE [1] and MB [ [4] for the derivations of the MB representations and for the $\varepsilon$-expansions. In section 2 we apply the MB-approach to the massive Bhabha vertex and box functions and extract their $\varepsilon$-poles. Section 3 contains the treatment of both the virtual $\varepsilon$-poles and the endpoint singularities from an unresolved, massless particles in a pentagon diagram of massive Bhabha scattering. The method may be generalized to more complex cases, including higher loop orders, but explicit evaluations become then more and more complicated.

\section{Simple $\varepsilon$-poles: Massive QED vertex and box}

We will set $m=1$, and $s, t$ are the usual Mandelstam variables. The QED vertex function has the $F$-form:

$$
F(s)=[X[2]+X[3]]^{2}+[-s] X[2] X[3]
$$


leading, without a continuation in $\varepsilon$, to a one-dimensional $\mathrm{MB}$ representation and a series over residues [2]:

$$
\begin{aligned}
V(s) & =\frac{1}{2 s \varepsilon} \frac{e^{\varepsilon \gamma_{E}}}{2 \pi i} \int_{-i \infty-1 / 2}^{-i \infty-1 / 2} d z(-s)^{-z} \frac{\Gamma^{2}(-z) \Gamma(-z+\varepsilon) \Gamma(1+z)}{\Gamma(-2 z)} \\
& =-\frac{e^{\varepsilon \gamma_{E}}}{2 \varepsilon} \sum_{n=0}^{\infty} \frac{s^{n}}{\left(\begin{array}{c}
2 n \\
n
\end{array}\right)(2 n+1)} \frac{\Gamma(n+1+\varepsilon)}{\Gamma(n+1)} .
\end{aligned}
$$

The complete series may be summed directly with Mathematica ${ }^{1}$, and the vertex becomes:

$$
V(s)=-\frac{e^{\varepsilon \gamma_{E}}}{2 \varepsilon} \Gamma(1+\varepsilon){ }_{2} F_{1}[1,1+\varepsilon ; 3 / 2 ; s / 4] .
$$

Alternatively, one may derive the $\varepsilon$-expansion by exploiting the well-known relation with harmonic numbers $S_{k}(n)=\sum_{i=1}^{n} 1 / i^{k}$ :

$$
\frac{\Gamma(n+a \varepsilon)}{\Gamma(n)}=\Gamma(1+a \varepsilon) \exp \left[-\sum_{k=1}^{\infty} \frac{(-a \varepsilon)^{k}}{k} S_{k}(n-1)\right] .
$$

The product $\exp \left(\varepsilon \gamma_{E}\right) \Gamma(1+\varepsilon)=1+\frac{1}{2} \zeta[2] \varepsilon^{2}+O\left(\varepsilon^{3}\right)$ yields expressions with zeta numbers $\zeta[n]$, and, taking all terms together, one gets a collection of inverse binomial sums ${ }^{2}$; the first of them is the IR divergent part:

$$
\begin{aligned}
V(s) & =\frac{V_{-1}(s)}{\varepsilon}+V_{0}(s)+\cdots \\
V_{-1}(s) & =\frac{1}{2} \sum_{n=0}^{\infty} \frac{s^{n}}{\left(\begin{array}{c}
2 n \\
n
\end{array}\right)(2 n+1)}=\frac{1}{2} \frac{4 \arcsin (\sqrt{s} / 2)}{\sqrt{4-s} \sqrt{s}} .
\end{aligned}
$$

This procedure applies similarly to the Bhabha box diagram [7]. We take for definiteness the $s$ channel scalar loop integral. The $F$-form is (again with $m=1$ ):

$$
F(s, t)=[X[2]+X[4]]^{2}+[-s] X[1] X[3]+[-t] X[2] X[4],
$$

and an MB representation is, after continuation to small $\varepsilon$, a sum of two terms:

$$
\begin{aligned}
B(s, t)= & \frac{(-s)^{-\varepsilon}}{2 s t} \frac{\Gamma[1+\varepsilon] \Gamma[-\varepsilon]^{2}}{\Gamma[-2 \varepsilon]} \frac{e^{\varepsilon \gamma_{E}}}{2 \pi i} \int_{-i \infty-7 / 16}^{+i \infty-7 / 16} d z_{1}(-t)^{-z_{1}} \frac{\Gamma^{3}\left[-z_{1}\right] \Gamma\left[1+z_{1}\right]}{\Gamma\left[-2 z_{1}\right]} \\
& +\frac{1}{t^{2}} \frac{1}{\Gamma[-2 \varepsilon]} \frac{e^{\varepsilon \gamma_{E}}}{(2 \pi i)^{2}} \int_{-i \infty-3 / 4}^{+i \infty-3 / 4} d z_{1}\left(\frac{s}{t}\right)^{z_{1}} \Gamma\left[-z_{1}\right] \Gamma\left[-2\left(1+\varepsilon+z_{1}\right)\right] \Gamma\left[1+z_{1}\right]^{2} \\
& \times \int_{-i \infty-7 / 16}^{+i \infty-7 / 16} d z_{2}(-t)^{-\varepsilon-z_{2}} \Gamma\left[-z_{2}\right] \frac{\Gamma\left[-1-\varepsilon-z_{1}-z_{2}\right]^{2}}{\Gamma\left[-2\left(1+\varepsilon+z_{1}+z_{2}\right)\right]} \Gamma\left[2+\varepsilon+z_{1}+z_{2}\right] .
\end{aligned}
$$

\footnotetext{
${ }^{1}$ The expression for $V(s)$ was also derived in [6]; see additionally [6]. [2].

${ }^{2}$ For the first four terms of the $\varepsilon$-expansion in terms of inverse binomial sums or of polylogarithmic functions, see
} 
Due to the pre-factors, $\Gamma[-\varepsilon]^{2} / \Gamma[-2 \varepsilon]=-2 / \varepsilon+2 \zeta[2] \varepsilon+O\left(\varepsilon^{2}\right)$ and $1 / \Gamma[-2 \varepsilon]=-2 \varepsilon+4 \gamma_{E} \varepsilon^{2}+$ $O\left(\varepsilon^{3}\right)$, only the first integral contributes to the first two terms of the $\varepsilon$-expansion,

$$
\left.B(s, t)=\left[\frac{1}{\varepsilon-\ln (-s)}\right] \frac{V_{-1}(t)}{(-s)}+\right)(\varepsilon),
$$

where $V_{-1}$ is from (2.6), and the IR divergency is:

$$
B_{-1}(s, t)=\frac{V_{-1}(t)}{(-s)} .
$$

We reproduce here the well-known fact that IR-divergences of vertices and boxes are algebraically related, see e.g. [3].

\section{Mixed virtual and real IR-singularities: massive Bhabha pentagon}

Things become more interesting for pentagon diagrams (se also [3, 8, 9]). We again use Bhabha scattering as an example. A compact $F$-form is:

$$
F\left(s, t, t^{\prime}, V_{2}, V_{4}\right)=\left(x_{2}+x_{4}+x_{5}\right)^{2}+[-s] x_{1} x_{3}+\left[-V_{4}\right] x_{3} x_{5}+[-t] x_{2} x_{4}+\left[-t^{\prime}\right] x_{2} x_{5}+\left[-V_{2}\right] x_{1} x_{4} .
$$

It exhibits a set of five invariants (out of a set of 10 scalar products at choice) describing the kinematics of a $2 \rightarrow 3$ process (here assuming a final state emission of an unresolved photon from an $s$ channel box diagram). The $s, t, t^{\prime}$ are the usual Mandelstam variables for the fermions in $e^{+} e^{-} \rightarrow e^{+} e^{-} \gamma$, and:

$$
V_{i}=2 p_{f_{i}} p_{\gamma}, \quad i=1, \ldots 4 .
$$

The $V_{i}$ are proportional to the energy of the potentially unresolved massless particle.

From a subsequent phase space integration, we have to expect endpoint singularities arising from terms proportional to $1 / V_{2} \sim 1 / E_{\gamma}$ and $1 / V_{4} \sim 1 / E_{\gamma}$, so we have to control, for a complete treatment of the IR-problem, not only the $\varepsilon$-expansion, but also the first terms of the $V_{2}, V_{4}$ expansions for small $V_{2}, V_{4}$.

In fact, the $F$-form (3.1), written here in its shortest form, depends on those two of the four $V_{i}$ which are related to the phase space of the given topology.

The MB-representation is a useful tool for that problem. For the IR limit, we may approximate $t^{\prime}=t$, and the scalar pentagon may be written as:

$$
I_{5}=\frac{-e^{\varepsilon \gamma_{E}}}{(2 \pi i)^{4}} \prod_{i=1}^{4} \int_{-i \infty+u_{i}}^{+i \infty+u_{i}} d z_{i}(-s)^{z_{2}}(-t)^{z_{4}}\left(-V_{2}\right)^{z_{3}}\left(-V_{4}\right)^{-3-\varepsilon-z_{1}-z_{2}-z_{3}-z_{4}} \frac{\prod_{j=1}^{12} \Gamma_{j}}{\Gamma_{0} \Gamma_{13} \Gamma_{14}}
$$

with $u_{i}=(-5 / 8,-7 / 8, \ldots)$ and with a normalization $\Gamma_{0}=\Gamma[-1-2 \varepsilon]$, and the other $\Gamma$-functions are:

$$
\Gamma_{1}=\Gamma\left[-z_{1}\right], \quad \Gamma_{2}=\Gamma\left[-z_{2}\right], \quad \Gamma_{3}=\Gamma\left[-z_{3}\right], \quad \Gamma_{4}=\Gamma\left[1+z_{3}\right]
$$




$$
\begin{aligned}
\Gamma_{5} & =\Gamma\left[1+z_{2}+z_{3}\right], \quad \Gamma_{6}=\Gamma\left[-z_{4}\right], \quad \Gamma_{7}=\Gamma\left[1+z_{4}\right], \quad \Gamma_{8}=\Gamma\left[-1-\varepsilon-z_{1}-z_{2}\right] \\
\Gamma_{9} & =\Gamma\left[-2-\varepsilon-z_{1}-z_{2}-z_{3}-z_{4}\right], \quad \Gamma_{10}=\Gamma\left[-2-\varepsilon-z_{1}-z_{3}-z_{4}\right], \\
\Gamma_{11} & =\Gamma\left[-\varepsilon+z_{1}-z_{2}+z_{4}\right], \quad \Gamma_{12}=\Gamma\left[3+\varepsilon+z_{1}+z_{2}+z_{3}+z_{4}\right]
\end{aligned}
$$

and, in the denominator:

$$
\Gamma_{13}=\Gamma\left[-1-\varepsilon-z_{1}-z_{2}-z_{4}\right], \quad \Gamma_{14}=\Gamma\left[-\varepsilon-z_{1}-z_{2}+z_{4}\right] .
$$

Leaving out here the details of derivation (see [3] for that), we just mention that we have to consider, after continuation in $\varepsilon$, eleven MB-integrals, being at most 4-dimensional (for $t^{\prime}=t$ ). The resulting IR-sensible part is:

$$
\begin{aligned}
I_{5}^{I R} & =I_{5}^{I R}\left(V_{2}\right)+I_{5}^{I R}\left(V_{4}\right), \\
I_{5}^{I R}\left(V_{i}\right) & =\frac{I_{-1}^{S}\left(V_{i}\right)}{\varepsilon}+I_{0}^{S}\left(V_{i}\right) .
\end{aligned}
$$

The $\varepsilon$-pole is again proportional to that of the vertex:

$$
\frac{I_{-1}^{s}\left(V_{i}\right)}{\varepsilon}=\frac{1}{2 s V_{i} \varepsilon} \sum_{n=0}^{\infty} \frac{(t)^{n}}{\left(\begin{array}{c}
2 n \\
n
\end{array}\right)(2 n+1)}=\frac{V_{-1}(t)}{s V_{i} \varepsilon}
$$

and:

$$
I_{0}^{s}\left(V_{i}\right)=\frac{1}{2 s V_{i}} \sum_{n=0}^{\infty} \frac{(t)^{n}}{\left(\begin{array}{c}
2 n \\
n
\end{array}\right)(2 n+1)}\left[-2 \ln \left(-V_{i}\right)-3 S_{1}(n)+2 S_{1}(2 n+1)\right]
$$

where we have to understand $\ln \left(-V_{i}\right)=\ln \left(V_{i} / s\right)+\ln \left[-(s+i \delta) / m^{2}\right]$. The series for $I_{0}^{s}\left(V_{i}\right)$ may be summed up in terms of polylogarithmic functions with the aid of Table 1 of Appendix D of [10], see also [3].

Equations (3.6) - (3.9) are the main physical result of the study. One may express the complete $I R$-divergent part of an amplitude with 5-point functions in terms of those expressions, subtract it from the complete, divergent amplitude and get a matrix element, which is integrable in four dimensions.

In the rest of this short write-up we would like to demonstrate why we have here besides the harmonic numbers $S_{1}(n)$ also those of the kind $S_{1}(2 n+1)$. As mentioned, the scalar 5-point function may be written as a sum of eleven MB-integrals after $\varepsilon$-continuation, before $\varepsilon$-expansion. Two types of them contribute to the IR-part (there are four such integrals [in an ad-hoc notations $\left.J_{3}, J_{4}, J_{7}, J_{9}\right]$, but with a symmetry $\left.V_{2} \leftrightarrow V_{4}\right)$. The first one is:

$$
\begin{aligned}
J_{7} & =-\frac{\left(V_{2} / s\right)^{2 \varepsilon}}{s V_{4}} \Gamma[-2 \varepsilon] \Gamma[1+2 \varepsilon] \frac{e^{\varepsilon \gamma_{E}}}{2 \pi i} \int_{-i \infty-5 / 8}^{+i \infty-5 / 8} d z(-t)^{-1-z} \frac{\Gamma[\varepsilon-z] \Gamma[2 \varepsilon-z] \Gamma[-z] \Gamma[1+z]}{\Gamma[2 \varepsilon-2 z]} \\
& =-\frac{\left(V_{2} / s\right)^{2 \varepsilon}}{s V_{4}} \frac{e^{\varepsilon \gamma_{E}} \varepsilon \sqrt{\pi}}{2^{2 \varepsilon}} \frac{\Gamma[-2 \varepsilon] \Gamma[2 \varepsilon] \Gamma[1+2 \varepsilon]}{\Gamma[3 / 2+\varepsilon]}{ }_{2} F_{1}[1,1+2 \varepsilon, 3 / 2+\varepsilon, t / 4]
\end{aligned}
$$




$$
=-\frac{\left(V_{2} / s\right)^{2 \varepsilon}}{s V_{4}} e^{\varepsilon \gamma_{E}} \Gamma[-2 \varepsilon] \Gamma[1+2 \varepsilon] \sum_{n=1}^{\infty} t^{n-1} \frac{\Gamma[\varepsilon+n] \Gamma[2 \varepsilon+n]}{\Gamma[2 \varepsilon+2 n]} .
$$

The $J_{7}$ is proportional to $1 / V_{4}$. We have a second integral of the same type, being proportional to $1 / V_{2}$ :

$$
J_{3}=J_{7}\left(V_{4} \leftrightarrow V_{2}\right)
$$

The other type of integrals $J_{4}, J_{9}$, with:

$$
J_{4}=J_{9}\left(V_{4} \leftrightarrow V_{2}\right)
$$

are two-fold MB-integrals:

$$
\begin{aligned}
J_{9}= & \frac{\Gamma[-2 \varepsilon]}{\Gamma[-1-2 \varepsilon]} \frac{e^{\varepsilon \gamma_{E}}}{(2 \pi i)^{2}} \int_{-i \infty-5 / 8}^{+i \infty-5 / 8} d z_{1} \int_{-i \infty-7 / 8}^{+i \infty-7 / 8} d z_{2}(-s)^{z_{2}}(-t)^{\varepsilon-z_{1}+z_{2}}\left(-V_{2}\right)^{-1-2 \varepsilon-z_{2}}\left(-V_{4}\right)^{-2-z_{2}} \\
& \times \Gamma\left[-z_{1}\right] \Gamma_{A}\left[-1-z_{2}\right] \Gamma\left[-2 \varepsilon-z_{2}\right] \Gamma\left[-1-\varepsilon-z_{1}-z_{2}\right] \Gamma_{C}\left[-\varepsilon+z_{1}-z_{2}\right] \Gamma\left[-z_{2}\right] \\
& \times \frac{\Gamma\left[2+z_{2}\right] \Gamma_{B}\left[1+2 \varepsilon+z_{2}\right] \Gamma\left[1+\varepsilon-z_{1}+z_{2}\right]}{\Gamma\left[-2 z_{1}\right] \Gamma\left[-1-2 \varepsilon-2 z_{2}\right]}
\end{aligned}
$$

The integral looks like being, in the limit $V_{4} \rightarrow 0$, too singular. This limit is an endpoint of the phase space integration. Let us close the contour to the left. We shift now the integration contour in $z_{2}$ to the left, raising in this way the (real part of the) power of $\left(-V_{4}\right)$ to a value which makes the photon phase space integral explicitely integrable at $V_{4} \rightarrow 0$ in $d=3-2 \varepsilon$ space dimensions. If singularities of the integrand (from $\Gamma$-functions) at some values $z_{2}=z_{R}$ are crossed one has to add the corresponding residues $J_{9}^{R}\left(z_{R}\right)$, so getting one-dimensional MB-integrals to be considered:

$$
J_{9}=2 \pi i \sum_{z_{R}} J_{9}^{R}\left(z_{R}\right)+J_{9}^{\text {shift }}
$$

The resulting integral $J_{9}^{\text {shift }}$ differs from $J_{9}$ only by the shifted integration path, but will now not contribute to the IR-singular part and will not be considered here any more. We see that only the residues of crossed singular points in $z_{2}$ contain the IR-relevant endpoint singularities in $V_{2}, V_{4}$. Here, two of them (at $z_{2}=-1$ [argument of $\Gamma_{A}$ in 3.13 ] and at $z_{2}=-1-2 \varepsilon$ [argument of $\Gamma_{B}$ in (3.13)]) contribute due to a shift from $\Re z_{2}=-7 / 8$ to $\Re z_{2}=-7 / 8-1=-15 / 8$. The first of them is:

$$
\begin{aligned}
J_{9 A}= & -\frac{\left(-V_{2}\right)^{-2 \varepsilon}}{s V_{4}} \frac{\Gamma[-2 \varepsilon] \Gamma_{B}[2 \varepsilon]}{\Gamma[-1-2 \varepsilon]} \frac{e^{\varepsilon \gamma_{E}}}{2 \pi i} \\
& \times \int_{-i \infty-5 / 8}^{+i \infty-5 / 8} d z_{1}(-t)^{-1-z_{1}} \frac{\left.\Gamma\left[-2 \varepsilon-z_{1}\right] \Gamma\left[-\varepsilon-z_{1}\right] \Gamma\left[-z_{1}\right] \Gamma_{C}\left[1+z_{1}\right]\right)}{\left.\Gamma[-1-2 \varepsilon] \Gamma\left[-2 \varepsilon-2 z_{1}\right)\right]} \\
= & \frac{\left(-V_{2}\right)^{-2 \varepsilon} 2^{2 \varepsilon}}{s V_{4}} e^{\varepsilon \gamma_{E}} \frac{\varepsilon \sqrt{\pi} \Gamma[-2 \varepsilon]^{2} \Gamma_{B}[2 \varepsilon]}{\Gamma[-1-2 \varepsilon] \Gamma[3 / 2-\varepsilon]} \quad{ }_{2} F_{1}[1,1-2 \varepsilon, 3 / 2-\varepsilon, t / 4] .
\end{aligned}
$$

We performed here an irrelevant shift $z_{1} \rightarrow z_{1}+\varepsilon$ in order to make the argument of $\Gamma_{C}$ independent of $\varepsilon$. This will be here the only $\Gamma$-function producing residues in $z_{1}$ when closing again the contour 
to the left. The explicit $\varepsilon$-expansion of the hypergeometric function in 3.15 ) may be obtained with the Mathematica package HypExp2 [11, 5]. Alternatively, we may perform the sum of residues arising from $\Gamma_{C}\left(1+z_{1}\right)$ directly:

$$
J_{9 A}=-\frac{\left(-V_{2}\right)^{-2 \varepsilon}}{s V_{4}} e^{\varepsilon \gamma_{E}} \frac{\Gamma[-2 \varepsilon] \Gamma[2 \varepsilon]}{\Gamma[-1-2 \varepsilon]} \sum_{n=1}^{\infty} t^{n-1} \frac{\Gamma[-2 \varepsilon+n] \Gamma[-\varepsilon+n]}{\Gamma[-2 \varepsilon+2 n]},
$$

and apply then (2.4) to it. It is here where we may see why the harmonic numbers $S_{1}(2 n+1)$ appear, which were not contributing to the vertex or the box: There was no $\Gamma$-function with an $\varepsilon$-shifted, doubled argument in the denominator of the final sums, while here this appears. In the general massive case, this will usually happen.

The second residue crossed by the contour shifting in the $z_{2}$-plane gives the third kind of contribution to be added; after a shift $z_{1} \rightarrow z_{1}-\varepsilon$ :

$$
\begin{aligned}
J_{9 B}= & \frac{\left(V_{4} / s\right)^{2 \varepsilon}}{s V_{4}} \Gamma[1-2 \varepsilon] \Gamma[-2 \varepsilon] \Gamma_{A}[2 \varepsilon] \frac{e^{\varepsilon \gamma_{E}}}{2 \pi i} \int_{-i \infty-5 / 8}^{+i \infty-5 / 8} d z_{1}(-t)^{-1-z_{1}} \\
& \times \frac{\Gamma\left[\varepsilon-z_{1}\right] \Gamma\left[2 \varepsilon-z_{1}\right] \Gamma\left[-z_{1}\right] \Gamma_{C}\left[1+z_{1}\right]}{\Gamma[-1-2 \varepsilon] \Gamma\left[2 \varepsilon-2 z_{1}\right]} \\
= & \frac{\left(V_{4} / s\right)^{2 \varepsilon}}{s V_{4}} e^{\varepsilon \gamma_{E}} \varepsilon \sqrt{\pi} \frac{\Gamma[1-2 \varepsilon] \Gamma[-2 \varepsilon] \Gamma[2 \varepsilon]^{2}}{2^{2 \varepsilon} \Gamma[-1-2 \varepsilon] \Gamma[3 / 2+\varepsilon]}{ }_{2} F_{1}[1,1+2 \varepsilon, 3 / 2+\varepsilon, t / 4] \\
= & \frac{\left(V_{4} / s\right)^{2 \varepsilon}}{s V_{4}} e^{\varepsilon \gamma_{E}} \frac{\Gamma[1-2 \varepsilon] \Gamma[-2 \varepsilon] \Gamma[2 \varepsilon]}{\Gamma[-1-2 \varepsilon]} \sum_{n=1}^{\infty} t^{n-1} \frac{\Gamma[\varepsilon+n] \Gamma[2 \varepsilon+n]}{\Gamma[2 \varepsilon+2 n]}
\end{aligned}
$$

Again, this may be expanded into an $\varepsilon$-series over inverse binomial sums by use of (2.4).

Collecting everything together, we rediscover (3.6) (plus additional terms of no relevance for the IR-treatment):

$$
J_{3}+J_{4 A}+J_{4 B}+J_{7}+J_{9 A}+J_{9 B}=\frac{1}{\varepsilon}\left[I_{-1}^{s}\left(V_{2}\right)+I_{-1}^{s}\left(V_{4}\right)\right]+I_{0}^{s}\left(V_{2}\right)+I_{0}^{s}\left(V_{4}\right)+\cdots
$$

\section{Conclusions}

We gave a pedagogical introduction to the treatment of mixed real and virtual IR-singularities. This kind of problems arises in NNLO problems, where one has to treat the unresolved massless particle phase space for loop integrals. The presented method was exemplified for a scalar integral, but it may be easily applied to general tensor functions. For the QED pentagon, this is discussed in [3], which may be considered as an introduction to this presentation. A derivation of MB-representations for higher $n$ point functions or for multi-loop integrals is more or less straightforward, although an analytical evaluation will become more and more troublesome. It is an interesting open question how useful the MB techniques might appear for realistic, so far unsolved applications.

\section{Acknowledgments}

We would like to thank J. Fleischer for useful discussions.

The present work is supported in part by the European Community's Marie-Curie Research Training Networks MRTN-CT-2006-035505 'HEPTOOLS' and MRTN-CT-2006-035482 'FLAVIAnet', 
and by Sonderforschungsbereich/Transregio 9-03 of Deutsche Forschungsgemeinschaft 'Computergestützte Theoretische Teilchenphysik'.

\section{References}

[1] J. Gluza, K. Kajda, and T. Riemann, AMBRE - a Mathematica package for the construction of Mellin-Barnes representations for Feynman integrals, Comput. Phys. Commun. 177 (2007) 879-893, [arXiv:0704.2423 [hep-ph]].

[2] J. Gluza, F. Haas, K. Kajda, and T. Riemann, Automatizing the application of Mellin-Barnes representations for Feynman integrals, PoS (ACAT) (2007) 081, arXiv:0707.3567 [hep-ph].

[3] J. Gluza and T. Riemann, New results for 5-point functions, Proc. LCWS, Hamburg (2007) [arXiv:0712.2969 [hep-ph]. See also at http://www-zeuthen.desy.de/ riemanns/LCWS07-Loops.htm.

[4] M. Czakon, Automatized analytic continuation of Mellin-Barnes integrals, Comput. Phys. Commun. 175 (2006) 559-571, hep-ph/0511200].

[5] T. Huber and D. Maitre, HypExp 2, expanding hypergeometric functions about half- integer parameters, arXiv:0708.2443 [hep-ph].

[6] A. Davydychev and M. Kalmykov, New results for the E-expansion of certain one-, two- and three-loop Feynman diagrams, Nucl. Phys. B605 (2001) 266-318, http://arXiv.org/abs/hep-th/0012189.

[7] J. Fleischer, J. Gluza, A. Lorca, and T. Riemann, First order radiative corrections to Bhabha scattering in d dimensions, Eur. J. Phys. 48 (2006) 35-52, [hep-ph/ 0606210 ].

[8] J. Fleischer. Application of Mellin-Barnes representation to the calculation of massive five-point functions in Bhabha scattering, talk given at the Conference on Frontiers in Perturbative Quantum Field Theory, June 14-16 2007, ZiF, Bielefeld.

[9] J. Fleischer, J. Gluza, K. Kajda, and T. Riemann, Pentagon diagrams of Bhabha scattering, Acta Phys. Polon. B38 (2007) 3529, [arXiv: 0710.5100 [hep-ph]].

[10] A. Davydychev and M. Kalmykov, Massive Feynman diagrams and inverse binomial sums, Nucl. Phys. B699 (2004) 3-64, hep-th/0303162].

[11] T. Huber and D. Maitre, HypExp, a Mathematica package for expanding hypergeometric functions around integer-valued parameters, Comput. Phys. Commun. 175 (2006) 122-144, [hep-ph/0507094]. 